\title{
Die Bedeutung der Falkenjagd für das Hofleben im Mittelalter
}

\author{
Anna Lena Eberl \\ Kernfach: Mittelalter \\ eingereicht bei: Ass.-Prof. MMag. ${ }^{\text {a }}$ Dr. ${ }^{\text {in }}$ Christina Antenhofer \\ eingereicht im: SS 2014 \\ Rubrik: PS-Arbeit
}

\begin{abstract}
The Importance of Falcons and Falconry for Court Life in the Middle Ages

This paper deals with the significance of falcons at medieval courts using the example of Albrecht III Achilles, Elector of Brandenburg. In a letter to his son-in-law Eberhard II, Duke of Württemberg, he has to deny the delivery of some falcons as Albrecht himself has no appropriate ones at his court. This paper examines why Falcons were important as valuable objects and gifts. Furthermore, it discusses how falconry could influence prestige and politics in the Middle Ages.
\end{abstract}

\section{Einleitung}

Auf vielen Wandgemälden und Abbildungen in Handschriften des Mittelalters sind adlige Damen und Herren mit Falken zu sehen. Dass diese Tiere von Bedeutung für das Hofleben dieser Zeit waren, zeigt sich u. a. auch daran, dass sie in der Minnelyrik des Öfteren aufgegriffen wurden. So schreibt etwa der Dichter Der von Kürenberg in der ersten Strophe seines „Falkenliedes“: „Ich zôch mir einen valken mêre danne ein jâr. / dô ich in gezamete, als ich in wolte hân, / und ich im sîn gevidere mit golde wol bewant, / er huop sich ûf vil hôhe und flouc in ándèriu lant." Obwohl es hier symbolisch um eine Liebesbeziehung geht und der Falke dabei für den Mann steht, der abhandenkommt und zu einer anderen entflieht, wird durch diese Strophe bereits eines deutlich: Die

1 Der von Kürenberg, Ich zôch mir einen valken, in: Minnesang. Mittelhochdeutsche Texte mit Übertragungen und Anmerkungen, hrsg. v. Helmut Brackert, Frankfurt am Main ${ }^{8} 2004$, S. 12 
Zähmung der Falken für den Hof und die Jagd dauerte oft sehr lange und die Tiere waren deshalb kostbar. Außerdem galten die Tiere offenbar als angesehen genug, um sie als Symbolfiguren in der Minnelyrik zu verwenden. Ausgehend von einem Brief, in dem die Bitte um einen Falken abgelehnt werden muss, wird in dieser Arbeit der Frage nachgegangen, welche Bedeutung diese Tiere und die Jagd mit innen für das Hofleben im Mittelalter hatten. Zudem soll erläutert werden, woher die Falken stammten und ob es üblich war, sie zu tauschen oder zu verschenken. Der Arbeit liegt die These zugrunde, dass Falken ein wichtiges und kostbares Prestigeobjekt waren und daher an keinem großen Hof fehlen durften und dass die Falkenjagd von Bedeutung für Ansehen und Politik der Adligen war.

Mit der Jagd im Mittelalter beschäftigen sich zahlreiche Publikationen, wenn auch die meisten davon inzwischen nicht mehr ganz aktuell sind. Die Falkenjagd selbst wird dabei aber häufig nur am Rande bzw. in einem Kapitel aufgegriffen. Zunächst sei diesbezüglich auf den Eintrag zur Beizjagd, also zur Jagd mit Vögeln, im Lexikon des Mittelalters verwiesen, der einen ersten geschichtlichen Überblick gibt, wie diese in Europa aufkam. ${ }^{2}$ Als Grundlage für das Thema der Falkenjagd dienen dieser Proseminararbeit mehrere Monografien und Sammelbände: Hervorzuheben wären dabei etwa die Diplomarbeit von Armin Stecher "Die Jagd im Mittelalter" aus dem Jahr 19963, die sich u. a. mit den einzelnen Falkenarten und deren Zucht auseinandersetzt, sowie der Sammelband „Jagd und höfische Kultur im Mittelalter" von 1997, herausgegeben von Werner Rösener, der mehrere Artikel zum Thema enthält. ${ }^{4}$ Außerdem wurde auf die Monographie „Im Gefolge Dianas. Frauen und höfische Jagd im Mittelalter (1200-1500)" von Katharina Fietze aus dem Jahr 2005 zurückgegriffen ${ }^{5}$, die sich insbesondere mit der Rolle der Frauen bei höfischen Jagdgesellschaften auseinandersetzt, aber auch ausführliche allgemeine Überlegungen zur Falkenjagd anstellt. Ähnliches gilt für das Werk von Barbara Hammes, „Ritterlicher Fürst und Ritterschaft. Konkurrierende Vergegenwärtigung ritterlich-höfischer Tradition im Umkreis südwestdeutscher Fürstenhöfe 1350-1450“6, aus dem Jahr 2011 , das Traditionen am Hofe behandelt und dabei auch auf die Jagd eingeht. Dezidiert mit der Falkenjagd setzt sich ein Sammelband zum Falkenbuch Friedrichs II. auseinander, der 2008 anlässlich einer Ausstellung zu diesem Thema entstanden ist. ${ }^{7}$ Zur Frage nach der Bedeutung der Tiere als Geschenke und Tauschobjekte liegt kaum Sekundärliteratur

2 Sigrid Schwenk, Beizjagd, in: Lexikon des Mittelalters, Band I: Aachen bis Bettelordenskirchen, MünchenZürich 1980, Sp. 1825-1826.

3 Armin Stecher, Die Jagd im Mittelalter, Dipl., Innsbruck 1996.

4 Zu nennen wären hierzu: Werner Rösener, Jagd, Rittertum und Fürstenhof im Hochmittelalter, in: Jagd und höfische Kultur im Mittelalter, hrsg. v. Werner Rösener, Göttingen 1997, S. 123-147; Joseph Morsel, Jagd und Raum. Überlegungen über den sozialen Sinn der Jagdpraxis am Beispiel des spätmittelalterlichen Franken, in: Ebd., S. 255-287; Klaus Militzer, Jagd und Deutscher Orden, in: Ebd., S. 325-363.

5 Katharina Fietze, Im Gefolge Dianas. Frauen und höfische Jagd im Mittelalter (1200-1500), Köln-WeimarWien 2005.

6 Barbara Hammes, Ritterlicher Fürst und Ritterschaft. Konkurrierende Vergegenwärtigung ritterlich-höfischer Tradition im Umkreis südwestdeutscher Fürstenhöfe 1350-1450, Stuttgart 2011.

7 Für diese Arbeit besonders interessant ist der sehr kurze, aber informative Artikel von Medrow. Lisa Anna Medrow, Falkenjagd im Mittelalter, in: Von der Kunst mit Vögeln zu jagen. Das Falkenbuch Friedrichs II. Kulturgeschichte und Ornithologie. Begleitband zur Sonderausstellung ,Kaiser Friedrich II. (1194-1250). Welt und Kultur des Mittelmeerraums' im Landesmuseum für Natur und Mensch Oldenburg, hrsg. v. Mamoun Fansa/Carsten Ritzau, Mainz am Rhein 2008, S. 18-20. 
vor. Einer der wenigen, der sich ausführlicher mit diesem Thema beschäftigt, ist Fritz Röhrig in seiner Monographie "Das Weidwerk"8 von 1933. Obwohl dieses Buch nicht aktuell ist, scheint es noch immer zu den Standardwerken zur (Beiz-)Jagd zu gehören, da sich der Großteil der eingesehenen Forschungsliteratur darauf bezieht. Deshalb soll es auch für diese Arbeit verwendet werden. Schließlich werden noch einige weitere Artikel und Monographien zitiert, die an dieser Stelle jedoch nicht thematisiert werden sollen, da sie für die vorliegende Arbeit keine zentrale Rolle spielen, sondern lediglich Zusatzinformationen liefern.

Im Folgenden soll zunächst ein Blick auf den Brief geworfen werden, der als Quelle und Ausgangspunkt für diese Arbeit dient. Es werden kurz die Biographien der beteiligten Personen sowie der Anlass des Schreibens erörtert, um einen Überblick darüber zu erhalten, wie es zur Bitte um einen Falken und deren Abweisung kam. Anschließend wird versucht, anhand der Aussagen des Briefes und mithilfe der Sekundärliteratur zu erläutern, welche Bedeutung die Falken sowie die Jagd mit innen für die Fürstenhöfe im Mittelalter hatte.

\section{Präsentation der Quelle}

Ausgangspunkt für diese Arbeit ist ein Schreiben von Kurfürst Albrecht von Brandenburg, das am 4. September 1482 verfasst wurde. Der Brief ist herausgegeben von Georg Steinhausen?, das Konzept dafür ist erhalten und liegt laut Herausgeber im Hausarchiv in Charlottenburg. Das Schreiben des Kurfürsten findet sich vollständig im Anhang dieser Arbeit.

Kurfürst Albrecht von Brandenburg, genannt Albrecht Achilles, schreibt an seinen Schwiegersohn, Graf Eberhard von Württemberg. Dieser hat ihn bereits zuvor (im August 1482) um die Zusendung von zwei oder zumindest einem Rotfalken gebeten, da er selbst in diesem Jahr viele Vögel verloren habe. ${ }^{10}$ Der Kurfürst antwortet inm nun, dass er momentan selber nicht so viele Falken habe, aber bald Rotfalken verfügbar wären. Sobald es so weit sei, werde er inm die vier schönsten davon zusenden. In seinem Brief schreibt Albrecht Achilles außerdem über den schlechten Zustand seiner Jagdvögel allgemein und den seiner Hunde sowie über einen Brief seines Sohnes Johann, dem er ebenfalls nichts schicken konnte.

Wo der Brief verfasst wurde, wird nicht angegeben. Da Albrecht von Brandenburg in Franken regierte, lässt sich jedoch vermuten, dass das Schreiben auch dort entstand.

Albrecht (1414-1486) war für eine ungewöhnlich lange Zeit politisch aktiv: Bereits 1440 wurde er als Hohenzoller Fürst des Unterlandes um Ansbach, ab 1470 war er auch

8 Fritz Röhrig, Das Weidwerk (Wald und Weidwerk in Geschichte und Gegenwart von Richard B. Hilf und Fritz Röhrig, Zweiter Teil), Potsdam 1933.

9 Georg Steinhausen (Hrsg.), Deutsche Privatbriefe des Mittelalters. 1. Band: Fürsten und Magnaten, Edle und Ritter (Denkmäler der deutschen Kulturgeschichte. Erste Abteilung Briefe. Erster Band Deutsche Privatbriefe des Mittelalters I.), Berlin 1899, Nr. 368, S. 248 f.

10 Der entsprechende Brief mit der Bitte an den Kurfürsten ist ebenfalls bei Georg Steinhausen ediert. Steinhausen, Deutsche Privatbriefe des Mittelalters, Nr. 367, S. 247 f. 
Kurfürst und Markgraf von Brandenburg. ${ }^{11}$ Der untersuchte Brief entstand gegen Ende seiner langen Regierungszeit. Während er in jungen Jahren viele Gefechte führte und in Streitigkeiten mit benachbarten Reichsgliedern verwickelt war - so stritt er z.B. mit der Reichsstadt Nürnberg um Rechte wie die Gerichtsbarkeit ${ }^{12}$ - war er als Kurfürst um die Erhaltung des Friedens bemüht. ${ }^{13}$ Wie für die meisten Adligen des Mittelalters spielten dabei auch für Albrecht Diplomatie und Heiratspolitik eine wichtige Rolle. Dementsprechend kümmerte er sich seit 1470 insbesondere um die politisch günstige Verehelichung seiner Töchter. ${ }^{14}$ Eine davon, Elisabeth von Brandenburg, heiratete schließlich Eberhard von Württemberg, an den der vorliegende Brief gerichtet wurde.

Interessant für diese Arbeit ist vor allem Albrechts höfisches Leben, da die Falkenzucht damit in engem Zusammenhang steht. Ernst Schubert verweist darauf, dass der Kurfürst schon von Kindheit an eine große Hofhaltung gewohnt war: "Schon in der Jugend hatte der Markgraf, dessen Vater einen ,König-Artus-Hof' im Stile des spätmittelalterlichen AdelsRomantizismus gehalten hatte, prägende Eindrücke fürstlicher Hofhaltung erfahren. “15 Dementsprechend hat auch Albrecht viele Turniere und Feste veranstaltet. Wie zahlreiche seiner Briefe zeigen, war der Kurfürst ein begeisterter Jäger: „Der Jagdleidenschaft ist Albrecht zeit seines Lebens nachgegangen. Immer wieder berichtet er in seinen Korrespondenzen von Jagderfolgen." ${ }^{16}$ Daher ist es auch selbstverständlich, dass an seinem Hof Falken und Jagdhunde, von denen in seinem Schreiben die Rede ist, stets vorhanden waren.

Der Empfänger des untersuchten Briefes ist Albrechts Schwiegersohn Eberhard VI. (14471504), Graf von Württemberg (seit 1496 als Herzog von Württemberg Eberhard II.). „Die Eheschließung (1465-1467) mit Markgräfin Elisabeth von Brandenburg [...] ordnete sich in die väterliche Politik ein: Ulrich V. und der Schwiegervater Albrecht Achilles waren die Exponenten der antipfälzischen bzw. antiwittelsbachischen Partei im Reich."17 Die Ehe verfolgte also politische Zwecke, verlief aber sehr unglücklich und blieb kinderlos. Für längere Zeit kehrte Elisabeth sogar zu ihrem Vater zurück. ${ }^{18}$ Wann dies genau der Fall war, konnte für diese Arbeit nicht herausgefunden werden. Da Albrecht in seinem Brief

11 Ernst Schubert, Albrecht Achilles, Markgraf und Kurfürst von Brandenburg (1414-1486), in: Fränkische Lebensbilder, hrsg. im Auftrag der Gesellschaft für fränkische Geschichtev. Gerhard Pfeiffer (Veröffentlichungen der Gesellschaft für Fränkische Geschichte, Reihe VII A: Fränkische Lebensbilder. Neue Folge der Lebensläufe aus Franken 4), Würzburg 1971, S. 130-172, hier S. 132.

12 Gabriel Zeilinger, Gruppenbild mit Markgraf. Albrecht ,Achilles' von Brandenburg (1414-1486), die Reichsfürsten seiner Zeit und die Frage nach zeitgenössischer und historiographischer Prominenz, in: Fürsten an der Zeitenwende zwischen Gruppenbild und Individualität. Formen fürstlicher Selbstdarstellung und ihre Rezeption (1450-1550). Wissenschaftliche Tagung Landeskulturzentrum Schloß Salzau, 27. -29. März 2008, hrsg. v. Oliver Auge/Ralf-Gunnar Werlich/Gabriel Zeilinger (Residenzenforschung. Herausgegeben von der Residenzen-Kommission der Akademie der Wissenschaften zu Göttingen, Band 22), Ostfildern 2009, S. 291-307, hier S. 293.

13 Schubert, Albrecht Achilles, S. 157.

14 Ebd., S. 162.

15 Ebd., S. 167.

16 Ebd., S. 168.

17 Dieter Stievermann, Eberhard VI./II., in: Das Haus Württemberg. Ein biographisches Lexikon, hrsg. v. Sönke Lorenz/Dieter Mertens/Volker Press, Stuttgart-Berlin-Köln 1997, S. 98-100, hier S. 98, Sp. 2.

18 Dieter Stievermann, Elisabeth, in: Das Haus Württemberg. Ein biographisches Lexikon, hrsg. v. Sönke Lorenz/Dieter Mertens/Volker Press, Stuttgart-Berlin-Köln 1997, S. 100, hier Sp. 2. 
Eberhard allerdings ganz unförmlich mit "Lieber sone“19, ohne die Angabe der Standestitel, anspricht, könnte man vermuten, dass die Beziehung zwischen den beiden zu dieser Zeit zumindest noch einigermaßen intakt war.

Elisabeth litt in ihrer Ehe vor allem auch unter den politischen Problemen ihres Mannes: Dieser stand in ständigem Konflikt mit seiner Familie, die die Landesteilung in Württemberg aufheben wollte und dies auch erreichte. Obwohl Eberhard 1496 schließlich dennoch zum Herzog ernannt wurde, wurde er einige Zeit später wieder entmachtet und musste fliehen. ${ }^{20}$ Insgesamt dürfte er also nicht sehr beliebt gewesen sein. Wie sein Schwiegervater Albrecht betrieb auch Eberhard ein sehr prunkvolles Hofleben. Von den Zeitgenossen wurde er daher oft als verschwenderisch und genusssüchtig beschrieben. ${ }^{21}$

Schließlich muss an dieser Stelle noch ein kurzer Blick auf Albrechts Sohn Johann geworfen werden, über den im Brief ausführlich geschrieben wird. Seine Biographie ist für diese Arbeit nicht von Interesse, allerdings ist jedoch ein Faktum zu erwähnen, das Aufschluss über die Aussagen des Kurfürsten gibt: Johann war in ständigen finanziellen Sorgen und verärgerte seinen Vater dadurch des Öfteren.22 Dies könnte unter Umständen ein Grund sein, warum Albrecht seinem Sohn keine Vögel für die Beizjagd zukommen ließ und im Schreiben über den Hund spottet, den ihm sein Sohn zur Verfügung stellen wollte.

Ausgehend vom Brief des Kurfürsten soll im Folgenden erläutert werden, welche Bedeutung Falken für den mittelalterlichen Hof hatten, und wie die Falkenjagd aussah.

\section{Falkenjagd und Falkenzucht am mittelalterlichen Hof}

Die Jagd war für das höfische Leben im Mittelalter von großer Bedeutung. Dementsprechend beschäftigen sich auch zahlreiche der bei Steinhausen edierten Briefe mit diesem Thema. Die Beizjagd macht dabei keine Ausnahme. Sie „erfreute sich beim mittelalterlichen Adel größter Beliebtheit. Schon Karl der Große war ein eifriger Beizjäger. “23 Im Frühmittelalter war die Jagd mit Vögeln vor allem im Osten sehr beliebt. Zur Zeit der Kreuzzüge verbreitete sie sich auch in Europa immer mehr. Von der Auseinandersetzung mit der Beizjagd zeugen verschiedene Quellen, u. a. die zahlreichen Falkentraktate oder das Falkenbuch Friedrichs II. mit dem Titel "De arte venandi cum avibus".24

Im Folgenden soll näher erläutert werden, welchen Stellenwert Falkenjagd und Falkenzucht an den deutschsprachigen mittelalterlichen Höfen einnahmen, und wie sie durchgeführt wurden.

19 Steinhausen, Deutsche Privatbriefe des Mittelalters, Nr. 368, S. 248.

20 Stievermann, Eberhard VI./II., S. 98/Sp. 1-S. 99/Sp. 2.

21 Ebd., S. 100/Sp. 1.

22 Cordula Nolte, Gendering Princely Dynasties: Some Notes on Family Structure, Social Networks, and Communication at the Courts of the Margraves of Brandenburg-Ansbach around 1500, in: Gender \& History 12 (2000), Heft 3, S. 704-721, hier S. 711.

23 Stecher, Jagd im Mittelalter, S. 70.

24 Schwenk, Beizjagd, Sp. 1826. 


\section{Die Falkenjagd als Gesellschaftssport}

Obwohl im ausgewählten Schreiben des Kurfürsten Albrecht nicht ausdrücklich geschildert wird, wofür die Falken gebraucht werden, ist doch klar, dass Eberhard um Jagdvögel gebeten hat. Immerhin muss Albrecht dessen Bitte um Falken mit den Worten abweisen, er habe „nit so vil falcken, das wir trostlich ein raiger konnen fahen“25. Außerdem beschreibt der Kurfürst, wie seine Vögel gemeinsam einen Reiher jagen. Es wird deutlich, dass die Fähigkeit zur Beizjagd über Wert bzw. Wertlosigkeit der Falken bestimmt I,die anndern sind nit wert, das mans falcken nennt"26). Es stellt sich nun die Frage, warum Eberhard überhaupt mit der Bitte um Falken an seinen Schwiegervater herantritt. Grundsätzlich gäbe es dafür zwei Möglichkeiten: Einerseits um damit jagen zu können, andererseits um sie als Geschenk zu verwenden. Auf den zweiten Aspekt wird in einem späteren Kapitel der Arbeit noch näher eingegangen.

Da die Beizjagd beim mittelalterlichen Adel sehr beliebt war, wurde sie auch an vielen Höfen durchgeführt, sofern es die Möglichkeit dazu gab. Abgerichtete Greifvögel verfolgten dabei das Wild (wildlebende Wasser- und Hühnervögel sowie kleineres Haarwild) auf offenen Landschaften wie Feldern, Wiesen oder Flussniederungen. ${ }^{27}$ Insbesondere die im Brief erwähnten Reiher waren dabei ein beliebtes Jagdziel. Unterschieden wird grundsätzlich zwischen zwei Arten von Beizjagden: Der Jagd in "niederem Flug”, bei der der Falke direkt auf die Beute geworfen wurde und dieser im Gleitflug folgte, sowie der Beize im „hohen Flug”, bei der hochfliegendes Flügelwild im offenen Gelände gejagt wurde, indem der Vogel in der Höhe kreiste und sich von dort im Sturzflug auf die Beute stürzte. ${ }^{28}$ Die Beizjagd selbst war für das höfische Publikum bzw. für die Adligen ein Spektakel, das gerne beobachtet wurde. Wichtig ist dabei zu erwähnen, dass sie nicht hauptsächlich dem Nahrungserwerb diente, sondern andere Aspekte wie Sport und Geschicklichkeit im Vordergrund standen. ${ }^{29}$ Auch Frauen nahmen zahlreich daran teil.

Die Falkenjagd war sehr kostspielig: Man brauchte ausgebildete Vögel, Pferde, um der Jagd zu folgen, entsprechendes Jagdpersonal und auch Hunde. Es ist nicht unlogisch, dass Kurfürst Albrecht in seinem Brief vom Bericht über seine Vögel auf die Jagdhunde übergeht. Immerhin waren im Mittelalter Hund und Vogel "die beiden komplementären Pole der Jagd schlechthin“30. Außerdem waren auch bei der Falkenjagd stets Hunde anwesend: Falls nötig, halfen diese dem Beizvogel, das Wild vollends zu Boden zu bringen, und spürten die erlegte Beute auf. ${ }^{31}$ Obwohl der Aufwand für die Fürsten bei der Jagd viel größer war als der Wert des erbeuteten Wildes, lohnte sich dieser dennoch aus einem bestimmten Grund. Die (Falken-)Jagd war sowohl Sport als auch gesellschaftliches Ereignis und als solches für die Politik der einzelnen Adligen wichtig:

25 Steinhausen, Deutsche Privatbriefe des Mittelalters, Nr. 368, S. 248.

26 Ebd.

27 Fietze, Im Gefolge Dianas, S. 3.

28 Ebd., S. 72.

29 Rösener, Jagd, S. 141.

30 Morsel, Jagd und Raum, S. 276.

31 Fietze, Im Gefolge Dianas, S. 72. 
„Trotz der echten Begeisterung und Leidenschaft, mit der viele Kaiser, unter innen Karl der Große, Friedrich II. und Maximilian I., der Jagd nachgingen, war diese niemals ausschließlich ein Freizeitvergnügen, sondern erfüllte repräsentative, integrierende und erzieherische Funktionen. “32

Mit der Ausrichtung einer Beizjagd konnte man seine Gastfreundlichkeit, Freigebigkeit und (durch besonders prachtvolle Falken) seinen Reichtum unter Beweis stellen sowie Kontakte knüpfen und pflegen. Das heißt, die Falkenjagd war für die Diplomatie und die Politik an den mittelalterlichen Höfen von großer Bedeutung, sowie vor allem auch für das Prestige der Adligen.

\section{Aufzucht und Zähmung von Falken für die Jagd}

Wie bereits erwähnt, ist bekannt, dass Albrecht von Brandenburg leidenschaftlicher Jäger war. Er schreibt auch in seinem Brief über seine Jagdhunde und die Angestellten, die diese betreuen. Da er aus dem hohen Adel stammte, ist es nur selbstverständlich, dass er nicht nur Falken, sondern auch Personal für diese an seinem Hof hatte. Wie Barbara Hammes erläutert, war für die württembergischen Fürsten die Jagd ebenfalls ein beliebter Zeitvertreib, weshalb an ihren Höfen immer auch Falkner zu finden waren. ${ }^{33}$ Graf Eberhard war nicht der erste seiner Familie, der sich mit der Bitte um Falken an einen anderen Adligen wandte. So ist zum Beispiel bei Steinhausen ein beigelegter Zettel zu einem Brief des Grafen Ulrich von Württemberg aus dem Jahr 1454 abgedruckt, in dem dieser Albrecht von Brandenburg ebenfalls um die Zusendung von Rotfalken bittet. ${ }^{34}$

Auf die einzelnen Vogel-/Falkenarten, mit denen gejagt wurde (wie z.B. der Sakerfalke, der Gerfalke etc.), wird an dieser Stelle nicht näher eingegangen, da diese für die Analyse des Briefes bzw. für die Fragestellung nicht relevant sind. Kurz erwähnt werden sollen jedoch der Rotfalke sowie der Blaufuß, da diese im Schreiben des Kurfürsten vorkommen.

Albrecht schreibt an Eberhard, dass er bald Rotfalken haben wird, von denen dieser vier bekommen sollte. Der Rotfalke ist einerseits zwar leicht zu zähmen, kann andererseits aber keine langen Flugzeiten bewältigen und lebt auch nicht so lange wie andere Falken. ${ }^{35}$ Dass er leicht zähmbar ist, kann erklären, wieso Albrecht bald mehrere Rotfalken zum Verschenken haben wird, während inm andere Beizvögel nicht zur Verfügung stehen. Im Brief ist außerdem die Rede von einem "fogelin“36, was Steinhausen mit "Blaufußvogel” übersetzt. Von diesem heißt es in der Literatur, dass er nicht so edel wie andere Falken sei, da ihm die nötige Kühnheit fehle - er bevorzugt es daher, nur kleines Federwild zu jagen. ${ }^{37}$ Vermutlich wird er aufgrund dieser Eigenschaften von Albrecht auch nicht als Falke bezeichnet.

32 Medrow, Falkenjagd, S. 18.

33 Hammes, Ritterlicher Fürst, S. 56.

34 Vgl. dazu: Steinhausen, Deutsche Privatbriefe des Mittelalters, Nr. 71, S. 55.

35 Stecher, Jagd im Mittelalter, S. 73.

36 Steinhausen, Deutsche Privatbriefe des Mittelalters, Nr. 368, S. 248.

37 Stecher, Jagd im Mittelalter, S. 73. 
Interessant sind diese Aussagen über die Falkenarten und deren Zähmbarkeit bzw. mangelnde Kühnheit deshalb, weil die Zucht und Abrichtung von Falken sehr schwierig und aufwendig war. An den mittelalterlichen Höfen gab es eigenes Personal (Falkner), das sich mit der Zähmung der Falken für die Jagd beschäftigte. Grundsätzlich konnte man sich die Vögel durch Fang oder Aufzucht beschaffen. ${ }^{38}$ Auch der Kauf von Falken war möglich, allerdings sehr kostspielig. Woher Albrecht von Brandenburg seine Beizvögel bezog, wird leider nicht erwähnt. Dass er bereits sicher vom baldigen Erhalt der Rotfalken weiß, könnte unterschiedliche Hintergründe haben: Wahrscheinlich ist, dass seine eigenen Falkner Vögel züchteten. Es könnte aber auch der Fall sein, dass auf seinen Ländereien (Jung-)Tiere gesichtet wurden, die man nun fangen wollte, oder aber ein befreundeter Adliger inm bereits die Zusendung von Falken versprochen hatte.

Fest steht, dass die Abrichtung der Vögel für die Jagd sehr schwierig, zeitaufwändig und damit auch kostspielig war. Junge Greifvögel mussten zunächst gezähmt und ausgebildet werden und sich an die Menschen und Jagdhunde gewöhnen. Anschließend richtete man sie für die Jagd auf eine bestimmte Wildart ab. ${ }^{39}$ Aus heutiger Sicht war die Falkenzähmung im Mittelalter ein grausames Unterfangen: Man ließ die Tiere hungern, vernähte innen die Augen, um sie gefügig zu machen (erst später wurden dafür Hauben verwendet), und entzog innen den Schlaf. Währenddessen trug man sie auf der Hand umher, um sie mit den Menschen vertraut zu machen. ${ }^{40}$ Bei der Zucht und Zähmung konnte es jedoch mitunter große Schwierigkeiten geben:

„Die empfindlichen Tiere galten als eigenwillig und waren zum Teil anfällig für bestimmte Krankheiten. Sie mußten abgerichtet, trainiert und veterinär versorgt werden. Die Abrichtung eines Beizvogels gelang nur bei intensiver, persönlicher Zuwendung. All das war Aufgabe von angestellten Berufsfalknern, den sog. Falkenieren, welche die Damen und Herren auch auf ihren Jagdausflügen begleiteten." ${ }^{\prime \prime 1}$

Das Training für die Jagd begann nach der Zähmung der Tiere. Die Falken wurden durch Appellübungen und Flugtraining auf Beuteattrappen für die Beize vorbereitet. ${ }^{42}$ Insgesamt nahm die Abrichtung der Vögel viel Zeit in Anspruch, und es gelang nicht immer, einen guten Falken für die Jagd heranzuziehen. Um so viele Beizvögel wie möglich für die Jagd zu erhalten, standen die dafür geeigneten Raubvögel unter dem Schutz der Fürsten und wurden entsprechend geschont. ${ }^{43}$ Dass sie schwer zu züchten und daher nicht im Übermaß vorhanden waren, erklärt, warum Albrecht angeben muss, momentan keine Falken hergeben zu können. Er verweist allerdings auf die schnellstmögliche Zusendung, was angesichts der Kostbarkeit der Beizvögel sehr aussagekräftig ist. Mit diesem Aspekt wird sich das folgende Kapitel näher beschäftigen.

38 Stecher, Jagd im Mittelalter, S. 74.

39 Fietze, Im Gefolge Dianas, S. 70.

40 Stecher, Jagd im Mittelalter, S. 75 f.

41 Fietze, Im Gefolge Dianas, S. 4.

42 Ebd., S. 71.

43 Röhrig, Weidwerk, S. 68. 


\section{Falken als Prestigeobjekte und wertvolle Geschenke}

Wie beschrieben, war die Beizjagd beim mittelalterlichen Adel sehr beliebt. Doch insbesondere die Jagd mit Edelfalken galt als vornehm und blieb daher dem Hochadel vorbehalten. ${ }^{44}$ Aufgrund ihrer aufwändigen Züchtung waren die Beizvögel sehr kostbar. Vor allem im Hochmittelalter wurden sie mit der zunehmenden Beliebtheit der Falkenjagd immer teurer, da der Bedarf an Tieren anstieg. Vielfach wurde dieser auch durch Importe abgedeckt.

„Neben dem einheimischen Wanderfalken, damals vielfach ,Pilgrimsfalke” genannt, wurden viele ausländische Falkenarten zur Beize verwendet. Es waren das in der Hauptsache der im südöstlichen Europa und in Asien beheimatete Saker und der aus Afrika und Südeuropa stammende Lanner. Am begehrtesten waren die nordischen Gerfalken, unter innen besonders der starke, hellbefiederte Isländer." ${ }^{45}$

All diese nicht einheimischen Falkenarten mussten jedoch zumeist teuer importiert werden, sofern sie nicht bereits am eigenen Hof vorhanden waren und gezüchtet werden konnten. Die nordischen Gerfalken waren beispielsweise nur als Geschenke erhältlich. ${ }^{46}$ Für alle anderen Beizvögel bestand die Möglichkeit, sie käuflich zu erwerben.

Im Laufe des Mittelalters entstand ein reger Handel mit Falken, v.a. aus den Niederlanden, Norwegen und Dänemark, der häufig von umherziehenden Falknern betrieben wurde, zuweilen schickten aber die Adligen auch eigene Händler für den Ankauf aus. Kaiser Maximilian hatte beispielsweise acht Falkner dafür abgestellt. ${ }^{47}$ Warum machte man sich aber nun die Mühe, Falken unterschiedlichster Art teuer von Händlern zu erwerben, obwohl es auch einheimische Tiere gab? Die Antwort darauf ist einfach: Falken galten als Statussymbole. ${ }^{48}$ Durch den Besitz von wertvollen Beizvögeln konnte man seinen eigenen Reichtum verdeutlichen. Nur wer genug Geld und Einfluss hatte, schaffte es, die edelsten Falken zu erhalten. Dass die Tiere ein Statussymbol, also ein Prestigeobjekt, für den Adel waren, zeigte sich $u$. a. auch an deren Ausrüstung: Häufig hielt man z.B. zwei Geschirre für sie bereit, eines für die Jagd, ein zweites, aufwändiges für die Repräsentation. ${ }^{49}$

Schließlich geht mit der Kostbarkeit der Falken und deren Prestigeträchtigkeit im Mittelalter noch ein weiterer Aspekt einher. Jagdfalken waren wichtige repräsentative Geschenke, vor allem wenn es um Politik ging. ${ }^{50}$ Wer sich die Gunst eines anderen Adligen sichern wollte, wählte häufig einen Beizvogel als Geschenk aus. Einerseits konnte man dadurch den eigenen Reichtum zeigen, andererseits durch die kostbare Gabe auch mitteilen, dass man für den anderen besondere Hochachtung oder Wertschätzung hegt.

44 Fietze, Im Gefolge Dianas, S. 69

45 Röhrig, Weidwerk, S. 66.

46 Gertrud Bauer, Maximilian I. und die Kunst, mit Vögeln zu jagen, Dipl., Innsbruck 1987, S. 4.

47 Röhrig, Weidwerk, S. 67.

48 Stecher, Jagd im Mittelalter, S. 71

49 Konrad Spindler, Falknerei in Archäologie und Geschichte unter besonderer Berücksichtigung der Beizjagd in Tirol, Innsbruck 1998, S. 10.

50 Stecher, Jagd im Mittelalter, S. 67 
Klaus Militzer beschäftigt sich in seinem Aufsatz „Jagd und Deutscher Orden“ mit dieser Thematik der Falkengeschenke. Der Deutsche Orden nahm dabei eine bedeutende Rolle im deutschsprachigen Raum ein. Er verteilte regelmäßig Beizvögel als Geschenke an hochgestellte Adlige, um diese für die eigenen Ziele zu gewinnen und sie sich generell gewogen zu halten. Von dieser Praxis des Deutschen Ordens profitierten sowohl geistliche als auch weltliche Fürsten. ${ }^{51}$ In den Quellen und der Forschungsliteratur sind zahlreiche Beispiele für die Falkengeschenke des Ordens dokumentiert. So ist etwa bekannt, dass die württembergischen Grafen im Herbst regelmäßig Falken vom Orden erhielten, ${ }^{52}$ und auch Kaiser Maximilian I. durfte sich über Beizvögel vom Hochmeister freuen, als Dank für den von inm gewährten Schutz. ${ }^{53}$ Da der Deutsche Orden selbst Tiere in einer Falkenschule züchtete und für die Jagd abrichtete, war er außerdem einer der Hauptlieferanten für den Handel. Gerade diese eigene Zucht und die großen Gewinne im Handel ermöglichten es aber, zahlreiche Vögel zu verschenken: Von der Falkenschule des Deutschen Ordens „erhielten 1401 der König von Böhmen, der König von Polen, der Herzog und die Herzogin von Österreich, der Herzog von Sachsen, der Erzbischof von Mainz Jagdfalken als Staatsgeschenke. 1480 gingen über 80 Falken an den Papst."54 Angesichts der Kostbarkeit der Tiere ist dies eine beachtliche Zahl.

Abschließend soll an dieser Stelle ein Bogen zurück zum Brief gemacht werden, von dem die Fragestellung der Arbeit ausging. Graf Eberhard bat seinen Schwiegervater Kurfürst Albrecht um die Zusendung von ein bis zwei Rotfalken, da er in diesem Jahr sehr viele verloren hatte. Da ein reger Handel und Tausch mit Falken bestand, war eine solche Bitte im Mittelalter nicht unüblich. Da Albrecht in seinem Brief keine Kosten oder Tauschobjekte nennt, kann vermutet werden, dass er die Beizvögel seinem Schwiegersohn als Geschenke zukommen lassen wird. Er schreibt, dass er Eberhard seine vier schönsten Rotfalken zusenden wird, sobald diese verfügbar sind. Auch wenn diese nicht zu den teuersten Beizvögeln gehörten - diese wären z.B. die nordischen Falken gewesen, die erst zu hohen Preisen importiert werden mussten -, kann es also als Zeichen von großer Wertschätzung angesehen werden, wenn Albrecht statt der gewünschten ein bis zwei Tiere sogar vier zur Verfügung stellte. Dadurch konnte der Kurfürst aber gleichzeitig seinen Reichtum und seine große Hofhaltung demonstrieren. Wofür Eberhard die Rotfalken verwenden möchte, lässt sich aus dem Schreiben nicht ableiten. Zu vermuten ist aber, dass er sie für die eigene Beizjagd sowie die Zucht einsetzen wollte.

Interessant an dieser Stelle ist auch, dass Albrecht selbst in seinem Brief an einer Stelle den Tauschhandel mit Falken anspricht. Er schreibt über den Briefwechsel mit seinem Sohn Johann unter anderem Folgendes: „Demnach haben wir seiner lieb kein falcken geschickt, so hat er unns auch kein konnen schicken."55 Dies alles zeigt, dass es auch innerhalb der einzelnen Familien üblich war, Falken zu verschenken und zu tauschen.

51 Militzer, Jagd und Deutscher Orden, S. 358.

52 Hammes, Ritterlicher Fürst, S. 57.

53 Bauer, Maximilian I., S. 73.

54 Stecher, Jagd im Mittelalter, S. 80 f.

55 Steinhausen, Deutsche Privatbriefe des Mittelalters, Nr. 368, S. 248. 


\section{Schluss}

Zusammenfassend lässt sich feststellen, dass die Falken und die Jagd mit innen für den mittelalterlichen Hof durchaus von Bedeutung waren. An den Höfen der Adligen, die es sich leisten konnten und ein entsprechend weites und offenes Gelände für die Jagd zur Verfügung hatten, fanden sich stets auch Vögel für die Beizjagd. Diese diente jedoch nicht dem Nahrungserwerb. Sie war einerseits vor allem als Sport sehr beliebt, andererseits war sie auch für die Diplomatie und Politik am Hof von Bedeutung. Durch die Ausrichtung einer Beizjagd konnte man seine Freigebigkeit sowie seinen Reichtum demonstrieren und gleichzeitig persönliche Kontakte knüpfen.

Da sich die Zucht von Falken schwierig und zeitaufwändig gestaltete, waren die Tiere kostbare Prestigeobjekte. Als solche wurden sie den Gästen am Hofe vorgezeigt, aber auch gehandelt und verschenkt. Besondere Statussymbole waren diejenigen Beizvögel, die aus anderen Ländern teuer importiert werden mussten. Mit dem Verschenken von Falken konnte man sich die Gunst anderer Adliger sichern sowie den eigenen Reichtum und die Wertschätzung dem Anderen gegenüber aufzeigen.

Der Brief des Kurfürsten Albrecht von Brandenburg an seinen Schwiegersohn Graf Eberhard VI. von Württemberg lässt in Hinblick auf die Bedeutung der Falken und der Beizjagden einige Punkte offen. Wir erfahren kaum etwas über die Falkenjagd und die Vogelzucht an Albrechts eigenem Hof und auch der Verwendungszweck der versprochenen Falken bleibt offen. Allerdings belegt der Brief an einigen Stellen eindeutig die Forschungsergebnisse: So wird etwa deutlich, dass die Falkenzucht ein schwieriges Unterfangen war und auch ein Hochadliger wie Albrecht, der eine große Jagdleidenschaft hatte, nicht immer genug gute Falken für die Beizjagd zur Verfügung hatte. Dies erklärt, warum die Tiere als so kostbar angesehen wurden. Außerdem erfahren wir aus dem Schreiben, dass der Tausch bzw. das Schenken von Vögeln für die Jagd durchaus eine übliche Praxis im Mittelalter war. Schließlich beschreibt Albrecht an einer Stelle seines Briefes auch, wie seine Falken gemeinsam einen Reiher jagen, und gibt damit Auskunft darüber, welches Wild bei der Beize verfolgt wurde - der Reiher war eines der beliebtesten Tiere dafür.

Dass Eberhard VI. und sein Schwiegervater den Falken bzw. der Jagd - in Albrechts Brief geht es auch um seine Jagdhunde - eine eigene Korrespondenz widmen, zeigt bereits, dass die Tiere für den mittelalterlichen Hof durchaus von Bedeutung waren. Der Adel wollte prachtvolle Falken besitzen, um seinen eigenen Status und Reichtum zu demonstrieren.

\section{Literatur}

Bauer, Gertrud, Maximilian I. und die Kunst, mit Vögeln zu jagen, Dipl. Innsbruck 1987.

Der von Kürenberg, Ich zôch mir einen valken, in: Minnesang. Mittelhochdeutsche Texte mit Übertragungen und Anmerkungen, hrsg. v. Helmut Brackert, Frankfurt am Main 82004, S. 12. 
Fietze, Katharina, Im Gefolge Dianas. Frauen und höfische Jagd im Mittelalter (12001500), Köln-Weimar-Wien 2005.

Hammes, Barbara, Ritterlicher Fürst und Ritterschaft. Konkurrierende Vergegenwärtigung ritterlich-höfischer Tradition im Umkreis südwestdeutscher Fürstenhöfe 1350-1450, Stuttgart 2011.

Medrow, Lisa Anna, Falkenjagd im Mittelalter, in: Von der Kunst mit Vögeln zu jagen. Das Falkenbuch Friedrichs II. - Kulturgeschichte und Ornithologie. Begleitband zur Sonderausstellung ,Kaiser Friedrich II. (1 194-1250). Welt und Kultur des Mittelmeerraums' im Landesmuseum für Natur und Mensch Oldenburg, hrsg. v. Mamoun Fansa/Carsten Ritzau, Mainz am Rhein 2008, S. 18-20.

Militzer, Klaus, Jagd und Deutscher Orden, in: Jagd und höfische Kultur im Mittelalter, hrsg. v. Werner Rösener, Göttingen 1997, S. 325-363.

Morsel, Joseph, Jagd und Raum. Überlegungen über den sozialen Sinn der Jagdpraxis am Beispiel des spätmittelalterlichen Franken, in: Jagd und höfische Kultur im Mittelalter, hrsg. v. Werner Rösener, Göttingen 1997, S. 255-287.

Nolte, Cordula, Gendering Princely Dynasties: Some Notes on Family Structure, Social Networks, and Communication at the Courts of the Margraves of Brandenburg-Ansbach around 1500, in: Gender \& History 12 (2000), Heft 3, S. 704-721.

Röhrig, Fritz, Das Weidwerk (Wald und Weidwerk in Geschichte und Gegenwart von Richard B. Hilf und Fritz Röhrig, Zweiter Teil), Potsdam 1933.

Rösener, Werner, Jagd, Rittertum und Fürstenhof im Hochmittelalter, in: Jagd und höfische Kultur im Mittelalter, hrsg. v. Werner Rösener, Göttingen 1997, S. 123-147.

Schubert, Ernst, Albrecht Achilles, Markgraf und Kurfürst von Brandenburg (1414-1486), in: Fränkische Lebensbilder, hrsg. v. Gerhard Pfeiffer /Veröffentlichungen der Gesellschaft für Fränkische Geschichte, Reihe VII A: Fränkische Lebensbilder. Neue Folge der Lebensläufe aus Franken 4), Würzburg 1971, S. 130-172.

Schwenk, Sigrid, Beizjagd, in: Lexikon des Mittelalters, Band I: Aachen bis Bettelordenskirchen, München-Zürich 1980, Sp. 1825-1826.

Spindler, Konrad, Falknerei in Archäologie und Geschichte unter besonderer Berücksichtigung der Beizjagd in Tirol, Innsbruck 1998.

Stecher, Armin, Die Jagd im Mittelalter, Dipl. Innsbruck 1996.

Steinhausen, Georg (Hrsg.), Deutsche Privatbriefe des Mittelalters. 1. Band: Fürsten und Magnaten, Edle und Ritter (Denkmäler der deutschen Kulturgeschichte. Erste Abteilung Briefe. Erster Band Deutsche Privatbriefe des Mittelalters I.), Berlin 1899.

Stievermann, Dieter, Eberhard VI./II., in: Das Haus Württemberg. Ein biographisches Lexikon, hrsg. v. Sönke Lorenz/Dieter Mertens/Volker Press, Stuttgart-Berlin-Köln 1997, S. 98-100. 
Stievermann, Dieter, Elisabeth, in: Das Haus Württemberg. Ein biographisches Lexikon, hrsg. v. Sönke Lorenz/Dieter Mertens/Volker Press, Stuttgart-Berlin-Köln 1997, S. 100.

Zeilinger, Gabriel, Gruppenbild mit Markgraf. Albrecht „Achilles' von Brandenburg (1414-1486), die Reichsfürsten seiner Zeit und die Frage nach zeitgenössischer und historiographischer Prominenz, in: Fürsten an der Zeitenwende zwischen Gruppenbild und Individualität. Formen fürstlicher Selbstdarstellung und ihre Rezeption (1450-1550). Wissenschaftliche Tagung Landeskulturzentrum Schloss Salzau, 27.-29. März 2008, hrsg. v. Oliver Auge/Ralf-Gunnar Werlich/Gabriel Zeilinger (Residenzenforschung. Herausgegeben von der Residenzen-Kommission der Akademie der Wissenschaften zu Göttingen 22), Ostfildern 2009, S. 291-307.

\section{Anhang}

Brief des Kurfürsten Albrecht von Brandenburg an seinen Schwiegersohn, Graf Eberhard von Württemberg, vom 4. September 1482:

„Lieber sone. Als ir unns geschriben habt umb bericht falcken, haben wir kaüm ein außkomen und nit so vil falcken, das wir trostlich ein raiger konnen fahen. Aber unns kommen rotfalcken. Sobald die kommen, wollen wir euch vier schicken, die schonsten, die darůnder sind. Unnser sune, marggraff Johanns, hat unns geschriben umb bericht falcken : was unnser bot vor uff dem wege, und schriben im auch darumb, wir hetten im nichts zu schicken, wir wollten dann selber gedarbt haben und nicht allein bedarbt, sunder nichts guts behalten. Wir haben ein fogelin [Blaufußvogel, Anm.] und drey falcken, die vergeen sich uber den bach, die anndern sind nit wert, das mans falcken nennt. Der anndern sind drey, sehen wir gern, das sie raiger fingen. Wenn wirs an zehen hetzen, so fahens kaüm ein. Ist der ein alt, der treibts umb ein weyl und dritt dann uff ein baüm, zu zeiten helffen die zween einannder, das sie ein raiger behalten, wie vor angezeigt ist. Demnach haben wir seiner lieb kein falcken geschickt, so hat er unns auch kein konnen schicken. Also hat er unns geschickt bey unnserm knecht vier wind, die zween sind ungeschaffen [häßlich, Anm.] und haben ein lauff, die zween sind hubscher art, noch jung, sie wollen aber noch nit die hasen erlauffen und sind itzund Jakobi jerig gewesen. Wir haben gar kein guten leidthund, sunder eytel welff [junge Hunde, Anm.] on zween, die haben fert [voriges Jahr, Anm.] gesucht. Hat yeder jeger ein an der handt, die suchen ser. Es ist in aber alles gerecht, was uff die payn kombt, so sind die jeger auch jung. Schreibt unns unnser sune, er woll unns ein leidthund schicken nach der brunfft, er sey aber sechs jar alt und vermog sich nit wol uff dem einen füß. Haben wir im wider geschriben, wenn wir in nicht uff die brünnt haben, das er dann sein alten betler selber behalt, wie wir unns jo sust durch das jar behelffen. Wir haben alt jegerknecht; sie werden unns wol hund berichten, das wir uff ein anndere brünft mit gots hilff húnd haben. Und darumb hab eur lieb ytzund ein mitleiden, dann wir haben sicher nichts in unnserm weidwerck, das zu loben sey, fur anders junger knecht. Drey ziehen wir auf, die lauffen ser, fert und heur hernach. Aber die jungen hund wollen sie nicht hinnach füren, als fert die alten theten. Datum am mitwoch nach Egidi anno etc. $82^{\circ}$.

(Adr.) An den von Wirtemberg. “56 
Anna Lena Eberl ist Studentin der Geschichte und der Germanistik im 4. Semester an der Universität Innsbruck. anna.I.eberl@student.uibk.ac.at

\section{Zitation dieses Beitrages}

Anna Lena Eberl, Die Bedeutung der Falkenjagd für das Hofleben im Mittelalter, in: historia. scribere 7 (2015), S. 223-236, [http://historia.scribere.at], 2014-2015, eingesehen 1.3.2015 (=aktuelles Datum).

(c) Creative Commons Licences 3.0 Österreich unter Wahrung der Urheberrechte der Autorlnnen. 